\title{
Computation with fuzzy quantities
}

\author{
Mirko Navara \\ Czech Technical University in Prague, Czech Republic
}

\begin{abstract}
Solving systems of equations (even linear ones) in standard fuzzy arithmetic may be a problem. We suggest an alternative approach which transfers the task to a linear space where the solution may be standard. Fuzzy intervals form a proper subset (in fact, a cone) in this linear space.
\end{abstract}

Keywords: Fuzzy number, fuzzy interval, fuzzy vector, fuzzy arithmetic, systems of linear equations

\section{Basic notions}

We deal with fuzzy subsets of the real line, $\mathbb{R}$. A fuzzy subset $A$ of $\mathbb{R}$ may be represented by its membership function $\mu_{A}: \mathbb{R} \rightarrow[0,1]$. Alternatively, $A$ can be determined by its collection of $\alpha$-cuts (sometimes called $\alpha$-level sets) ${ }^{\alpha} A=\left\{x \in \mathbb{R}: \mu_{A}(x) \geq \alpha\right\}$ for all $\alpha \in(0,1]$. By a fuzzy quantity (fuzzy inter$v a l)$ we mean a fuzzy subset $A$ of $\mathbb{R}$ satisfying the following three conditions [2]:

1. convexity and closedness: for each $\alpha \in(0,1]$, the $\alpha$-cut ${ }^{\alpha} A$ is a closed interval,

2. boundedness: the support of $A$, Supp $A=\{x \in$ $\left.\mathbb{R}: \mu_{A}(x)>0\right\}$, is bounded,

3. normality: the core,${ }^{1} A$, is nonempty.

If, moreover, the core of $A$ is a singleton, we call $A$ a fuzzy number.

In particular, a trapezoidal fuzzy interval $A=$ $\langle a, b, c, d\rangle$, where $a \leq b \leq c \leq d$, has a piecewise linear membership function

$$
\mu_{A}(x)= \begin{cases}\frac{x-a}{b-a} & \text { when } x \in[a, b), \\ 1 & \text { when } x \in[b, c], \\ \frac{d-x}{d-c} & \text { when } x \in(c, d], \\ 0 & \text { otherwise; }\end{cases}
$$

If, moreover, $b=c$, we speak of a triangular fuzzy number $\langle a, b, d\rangle$. Another special case is a crisp interval, $[a, d]=\langle a, a, d, d\rangle$, which has all cuts equal (except for the 0-cut which we do not consider here). A crisp real number $r$ is a special case of all the above and we shall identify it with the singleton: $r=[r, r]=\langle r, r, r\rangle=\langle r, r, r, r\rangle$.

The mapping $h_{A}:(0,1] \rightarrow \exp \mathbb{R}$, defined by $h_{A}(\alpha)={ }^{\alpha} A$, determines $A$ uniquely, giving the horizontal representation of $A$ [2]. (To emphasize the difference, we speak of the representation of a fuzzy set by the mapping $\mu_{A}: \mathbb{R} \rightarrow[0,1]$ as the vertical representation.)

\section{Standard fuzzy arithmetic and its properties}

The basic aim of fuzzy arithmetic is to extend the operations $+,-, \cdot, /$ to fuzzy quantities. Let $\square \in$ $\{+,-, \cdot, /\}$. In the standard fuzzy arithmetic (SFA), the operation $\square$ is extended to fuzzy intervals $A, B$ in the following two equivalent ways $[3,4]$ :

1. in the vertical representation by the extension principle

$$
\mu_{A \square B}(c)=\sup _{a \square b=c} \min \left\{\mu_{A}(a), \mu_{B}(b)\right\},
$$

2. in the horizontal representation

$$
\left.{ }^{\alpha}(A \square B)=\left\{a \square b \mid a \in{ }^{\alpha} A, b \in{ }^{\alpha} B\right)\right\} .
$$

Due to the proper definition of a fuzzy quantity, these two formulas coincide. This is important because (necessarily approximate) computing in the vertical representation requires extensive evaluation within two nested cycles, while computing in the horizontal representation uses the standard interval arithmetic for each cut. This is performed by the formulas

$$
\begin{aligned}
& {[a, b]+[c, d] }=[a+c, b+d], \\
& {[a, b]-[c, d] }=[a-d, b-c], \\
& r \cdot[a, b]=[\min (r a, r b), \max (r a, r b)] \\
&=\left\{\begin{aligned}
{[r a, r b] } & \text { if } r \geq 0, \\
{[r b, r a] } & \text { if } r<0,
\end{aligned}\right. \\
& {[a, b] \cdot[c, d]=} {[\min (a c, a d, b c, b d),} \\
&\max (a c, a d, b c, b d)], \min (a / c, a / d, b / c, b / d), \\
& {[a, b] /[c, d]=[\max (a / c, a / d, b / c, b / d)], }
\end{aligned}
$$

provided that the divisor $[c, d]$ does not contain 0 . (In this case, the result of division is still defined by the formula for the vertical representation, but it is not a fuzzy quantity.) The formulas for multiplication and division can be simplified when the fuzzy quantities are positive.

\section{Problems of standard fuzzy arithmetic}

Addition, resp. multiplication, of fuzzy quantitites has a neutral element 0 , resp. 1 . They are commutative and associative and they satisfy cancellativity:

$$
\begin{array}{rll}
A+B=A+C & \Longrightarrow & B=C, \\
A \cdot B=A \cdot C & \Longrightarrow & B=C .
\end{array}
$$


(In the latter formula, we must assume that $\mu_{A}(0)=$ 0.)

Addition and multiplication by a positive scalar satisfy the properties of operations in a linear space. Thus the equation

$$
r \cdot X=B
$$

where $r \neq 0$ and a fuzzy quantity $B$ are given and $X$ is the unknown fuzzy quantity, has a unique solution

$$
X=\frac{1}{r} \cdot B .
$$

However, some basic properties of operations with reals are not valid for fuzzy quantities. In particular, the following property of linear spaces

$$
(r+s) \cdot A=r \cdot A+s \cdot A
$$

may be violated if the signs of $r, s$ are not equal. It is even usual that

$$
\begin{gathered}
A-A \neq 0, \\
A / A \neq 1,
\end{gathered}
$$

etc. Counterexamples can be found already in interval arithmetic, e.g.

$$
[1,2]-[1,2]=[-1,1]
$$

This causes problems in solution of (systems of) equation, even when they are linear.

\section{Crucial example}

We shall show a detailed solution to a seemingly trivial equation. It demonstrates the difficulties encountered in computing with fuzzy quantities. Solvability will be discussed in detail. Later on, it will appear that more difficult problems can be transformed to this situation using our new approach and standard tools of linear algebra.

Example 1 The simplest linear equation

$$
X+A=B
$$

for fuzzy quantities cannot be solved by subtracting A from both sides:

$$
X+A-A=B-A
$$

because the left-hand side need not be equal to $X$. The solution within fuzzy quantities need not exist.

Let us first restrict to the case of usual interval arithmetic. A special form of (1),

$$
X+\left[a_{\ell}, a_{u}\right]=\left[b_{\ell}, b_{u}\right],
$$

has a unique solution within fuzzy quantities, the interval

$$
X=\left[b_{\ell}-a_{\ell}, b_{u}-a_{u}\right],
$$

iff

$$
\left(b_{u}-a_{u}\right)-\left(b_{\ell}-a_{\ell}\right) \geq 0 .
$$

The solvability condition is an inequality of the interval lengths:

$$
\left(b_{u}-b_{\ell}\right)-\left(a_{u}-a_{\ell}\right) \geq 0 .
$$

Otherwise, no solution exists.

In the general case, the $\alpha$-cuts of $A, B, X$ are functions of $\alpha$,

$$
\begin{aligned}
{ }^{\alpha} X & =\left[x_{\ell}(\alpha), x_{u}(\alpha)\right], \\
{ }^{\alpha} A & =\left[a_{\ell}(\alpha), a_{u}(\alpha)\right], \\
{ }^{\alpha} B & =\left[b_{\ell}(\alpha), b_{u}(\alpha)\right],
\end{aligned}
$$

where the lower bounds are non-decreasing and upper bounds are non-increasing functions. As before, we obtain only possible candidate for the solution, with bounds

$$
\begin{aligned}
x_{\ell}(\alpha) & =b_{\ell}(\alpha)-a_{\ell}(\alpha), \\
x_{u}(\alpha) & =b_{u}(\alpha)-a_{u}(\alpha) .
\end{aligned}
$$

We have to check whether there exists a fuzzy quantity with $\alpha$-cuts bounded by these functions.

One set of necessary conditions are the inequalities of interval lengths

$\left(b_{u}(\alpha)-b_{\ell}(\alpha)\right)-\left(a_{u}(\alpha)-a_{\ell}(\alpha)\right) \geq 0$ for all $\alpha \in(0,1]$.

These are not sufficient. It remains to check whether $x_{\ell}$ is non-decreasing and $x_{u}$ non-increasing. If $A, B$ are trapezoidal fuzzy numbers, the bounds of their $\alpha$-cuts are linear functions of $\alpha$ and it suffices to verify monotonicity for arbitrary two values of $\alpha$. In general, the procedure may be more difficult.

Let us finally demonstrate that the problem with monotonicity may occur in practice. Let us consider triangular fuzzy numbers $A=\langle 0,0,1\rangle, B=\langle 0,1,1\rangle$. Then the bounds are

$$
\begin{aligned}
a_{\ell}(\alpha) & =0, \\
a_{u}(\alpha) & =1-\alpha, \\
b_{\ell}(\alpha) & =\alpha, \\
b_{u}(\alpha) & =1 .
\end{aligned}
$$

This gives

$$
\begin{gathered}
x_{\ell}(\alpha)=b_{\ell}(\alpha)-a_{\ell}(\alpha)=\alpha, \\
x_{u}(\alpha)=b_{u}(\alpha)-a_{u}(\alpha)=\alpha,
\end{gathered}
$$

and although all intervals $\left[x_{\ell}(\alpha), x_{u}(\alpha)\right]$ are nonemtpy (singletons $\{\alpha\}$ ), they are not ordered by inclusion and there is no fuzzy quantity with such a system of $\alpha$-cuts.

Similarly, the equation

$$
X-C=B
$$

cannot be solved using

$$
X-C+C=B+C
$$

and need not have a solution. 
Remark 2 An alternative way of computation with fuzzy quantities, so-called constrained fuzzy arithmetic (CFA) does not suffer these problems, see [3, 4]. It is a totally different way of computation with imprecision describing other phenomena, thus these two apporaches cannot substitute each other. Besides, it is computationally much more demanding [6].

The solution of systems of linear equations in standard fuzzy arithmetic becomes much more difficult. In the sequel, we present another view on this problem and offer a method that allows to solve such tasks using as much of standard linear space techniques as possible. We shall demonstrate it on the most trivial examples (where its efficiency is not remarkable), but the same principles can be applied to arbitrarily complex systems of linear equations (where the contribution is significant).

\section{Inspiration: Free vectors in geometry and physics}

Fuzzy quantities form a commutative cancellative monoid which can be embedded into a linear space. This procedure follows that which allows to derive rational numbers as classes of pairs of integers with an equivalence $(a, b) \equiv(c, d)$ iff $a d=b c$. As we start from a continuum with an addition here, a better analogy is obtained from the construction of free vectors used in physics, as described below.

Let us consider an $n$-dimensional Euklidean space, $\mathcal{E}$. We define an equivalence relation $\equiv$ on $\mathcal{E}^{2}$ such that $(A, B) \equiv(C, D)$ iff $A, B, D, C$ (in this order) form a (possibly degenerated) parallelogram. This is equivalent to the condition that the centers of line segments $A D, B C$ coincide. For $(A, B) \in \mathcal{E}^{2}$, we denote by

$$
[(A, B)]=\left\{(C, D) \in \mathcal{E}^{2} \mid(A, B) \equiv(C, D)\right\}
$$

the respective equivalence class, called a free vector on $\mathcal{E}$, and by $\mathcal{E}^{2} / \equiv$ the collection of all free vectors on $\mathcal{E}$. (Alternatively, a free vector $[(A, B)]$ may be interpreted as the translation $\mathcal{E} \rightarrow \mathcal{E}$ which maps $A$ onto $B$.) If we fix an origin, $O \in \mathcal{E}$, a point $A \in \mathcal{E}$ may be identified with its position vector, $\vec{A}=[(O, A)] \in \mathcal{E}^{2} / \equiv$, and $\vec{A}=\vec{A}-\vec{O}$. Notice that the following conditions are equivalent:

- $(A, B) \equiv(C, D)$,

- $[(A, B)]=[(C, D)]$,

- $\vec{A}+\vec{D}=\vec{B}+\vec{C}$.

Remark 3 Instead of the whole $\mathcal{E}^{2}$, it is sufficient to start from a generating cone $\mathcal{C}$ in $\mathbb{R}^{2}$, i.e., $\mathcal{C} \varsubsetneqq \mathbb{R}^{2}$ such that

- $\vec{A} \in \mathcal{C}, \vec{B} \in \mathcal{C} \Longrightarrow \vec{A}+\vec{B} \in \mathcal{C}$,

- $\vec{A} \in \mathcal{C}, r \geq 0 \Longrightarrow r \cdot \vec{A} \in \mathcal{C}$,

- $\mathbb{R}^{2}=\mathcal{C}-\mathcal{C}=\{\vec{A}-\vec{B} \mid \vec{A} \in \mathcal{C}, \vec{B} \in \mathcal{C}\}$.

Then $\mathcal{C}^{2} / \equiv=\mathcal{E}^{2} / \equiv$.

\section{Fuzzy quantitites as vectors}

Using the idea of free vectors, we embedd fuzzy quantities into a linear space.

Definition 4 Let us denote by $\mathcal{I}$ the set of all fuzzy quantities. We define the relation $\equiv$ on $\mathcal{I}^{2}$ such that

$$
(A, B) \equiv(C, D) \text { iff } A+D=B+C .
$$

Proposition 5 The relation $\equiv$ is an equivalence which splits $\mathcal{I}^{2}$ to classes of equivalent couples of fuzzy quantities.

Definition 6 For $(A, B) \in \mathcal{I}^{2}$, we denote by

$$
[(A, B)]=\left\{(C, D) \in \mathcal{I}^{2} \mid(A, B) \equiv(C, D)\right\}
$$

the respective equivalence class and call it a free fuzzy quantity. The collection of all free fuzzy quantities will be denoted by $\mathcal{F}=\mathcal{I}^{2} / \equiv$.

Notice that, for all $A, B, E \in \mathcal{I}$,

$$
[(A, B)]=[(A+E, B+E)] .
$$

As a factor space of a linear space, $\mathcal{F}$ is equipped with the inherited linear operations. To distinguish them from operations with fuzzy quantities, we denote by $\oplus$ the vector addition in $\mathcal{F}$ and by $\odot$ the multiplication of vectors from $\mathcal{F}$ by scalars. Explicitly,

$$
[(A, B)] \oplus[(C, D)]=[(A+C, B+D)] .
$$

From this formula, multiplication by natural numbers follows and it can be extended by the standard mechanism to positive rational numbers and by continuity to positive real numbers:

$$
r \odot[(A, B)]=[(r \cdot A, r \cdot B)] \text { for } r \geq 0 .
$$

Nevertheless, multiplication by negative numbers is more complex. Suppose, e.g., that

$$
[(C, D)]=(-1) \cdot[(A, B)] .
$$

This means that

$$
\begin{aligned}
{[(A+C, B+D)] } & =[(A, B)] \oplus[(C, D)] \\
& =1 \cdot[(A, B)] \oplus(-1) \cdot[(A, B)] \\
& =(1-1) \cdot[(A, B)]=[(0,0)] .
\end{aligned}
$$

Unless $A, B$ are crisp numbers, we cannot choose $C=-A$ and $D=-B$ and there are no $C, D \in \mathcal{I}$ satisfying

$$
\begin{aligned}
& A+C=0, \\
& B+D=0 .
\end{aligned}
$$

Nevertheless, we shall derive another element of $\mathcal{F}$ which will play the role of $(-1) \cdot[(A, B)]$. 
Addition induces subtraction of vectors in $\mathcal{F}$, which will be denoted by $\ominus$. Vector

$$
[(T, U)]=[(A, B)] \ominus[(C, D)]
$$

should be a solution to the equation (in $\mathcal{F}$ )

$$
[(T, U)] \oplus[(C, D)]=[(A, B)],
$$

where $T, U \in \mathcal{I}$ are unknown fuzzy quantities. These cannot be determined by the equations

$$
\begin{aligned}
& T+C=A, \\
& U+D=B,
\end{aligned}
$$

which need not have solutions in $\mathcal{I}$ (see Example 1). Instead of that, we need another representative of the equivalence class. We may express the righthand side of (3) as

$$
[(A, B)]=[(A+C+D, B+C+D)]
$$

and

$$
[(T, U)] \oplus[(C, D)]=[(A+C+D, B+C+D)]
$$

leads to the system

$$
\begin{aligned}
& T+C=A+C+D, \\
& U+D=B+C+D
\end{aligned}
$$

with a unique solution

$$
\begin{aligned}
& T=A+D, \\
& U=B+C .
\end{aligned}
$$

The result

$$
[(T, U)]=[(A+D, B+C)]
$$

is a unique element of $\mathcal{F}$, but it has other equivalent forms, e.g.

$$
[(T, U)]=[(A+D+E, B+C+E)]
$$

for any $E \in \mathcal{I}$. We may correctly define

$$
[(A, B)] \ominus[(C, D)]=[(A+D, B+C)] .
$$

Let us summarize the operations derived above:

Theorem 7 The space $\mathcal{F}$ is equipped with the following operations:

$$
\begin{aligned}
{[(A, B)] \oplus[(C, D)] } & =[(A+C, B+D)], \\
{[(A, B)] \ominus[(C, D)] } & =[(A+D, B+C)], \\
r \odot[(A, B)] & = \begin{cases}{[(r \cdot A, r \cdot B)]} & \text { if } r \geq 0, \\
{[((-r) \cdot B,(-r) \cdot A)]} & \text { if } r<0 .\end{cases}
\end{aligned}
$$

It is a real linear space with operations $\oplus, \odot$ and zero vector $[(0,0)]$.

Definition 8 Unary minus $\ominus: \mathcal{F} \rightarrow \mathcal{F}$ is defined as

$$
\ominus[(C, D)]=[(0,0)] \ominus[(C, D)]=[(D, C)] .
$$

It can be interpreted also as multiplication by $(-1)$,

$$
\ominus[(C, D)]=(-1) \odot[(C, D)]
$$

Proposition 9 Properties of unary minus $\ominus$ on $\mathcal{F}$ :

1. $\ominus(\ominus[(C, D)])=[(C, D)]$,

2. $[(A, B)] \oplus(\ominus[(C, D)])=[(A, B)] \ominus[(C, D)]$,

3. $[(A, B)] \ominus(\ominus[(C, D)])=[(A, B)] \oplus[(C, D)]$,

4. $\ominus(r \odot[(C, D)])=(-r) \odot[(C, D)]=r \odot$ $(\ominus[(C, D)])$.

Definition 10 For each $A \in \mathcal{I}$, we define $\vec{A}=$ $[(0, A)] \in \mathcal{F}$.

Theorem 11 The mapping $e: A \mapsto \vec{A}$ preserves addition and multiplication by positive scalars, i.e.,

Proposition 12 1. $\vec{A} \in \mathcal{I}, \vec{B} \in \mathcal{I} \Longrightarrow \overrightarrow{A+B}=$ $\vec{A} \oplus \vec{B}$,

2. $\vec{A} \in \mathcal{I}, r \geq 0 \Longrightarrow \overrightarrow{r \cdot A}=r \odot \vec{A}$.

In particular, for $A=0$ we obtain $\overrightarrow{0}=[(0,0)]$, the zero vector in $\mathcal{F}$.

The set $\{(0, A) \mid A \in \mathcal{I}\}$ is a generating cone in $\mathcal{I}^{2}$.

Thus linear equations for fuzzy quantities can be translated in terms of the linear space $\mathcal{F}$, where they can be solved easily. However, this procedure is trivial only for systems of linear equations with positive coefficients. Negative coefficients and subtraction require a different treatment which will be discussed later on.

\section{Solvability}

The solution of a system of linear equations can be computed in the linear space $\mathcal{F}$ using standard procedures of linear algebra. However, only some of its elements correspond to fuzzy quantities from $\mathcal{I}$. The others do not have such a natural interpretation.

Example 13 Equation (1) from Example 1 can be rewritten as

$$
[(0, X)] \oplus[(0, A)]=[(0, B)]
$$

or

$$
\vec{X}+\vec{A}=\vec{B} .
$$

We immediately get the solution in $\mathcal{F}$ :

$[(0, X)]=[(0, B)] \ominus[(0, A)]=[(A, B)]=\vec{B} \ominus \vec{A}$.

In order to find a fuzzy quantity $X$ satisfying the latter equation, we have to return again to equation (1) and solve it as in Example 1.

In this trivial example, the new approach did not simplify our task, it only gave it another interpretation. In fact, $[(A, B)]$ denotes exactly the class 
solving equation (1) in $\mathcal{F}$. The question if this solution corresponds to some fuzzy quantity (i.e., if it belongs to the corresponding cone $e(\mathcal{I})$ ) does not have a trivial answer, as we saw in Example 1. The good news is that this procedure is the only technique required except for the standard solution of systems of linear equations. (These are the same in all linear spaces.) Thus the only added effort is at the end of the procedure, when we have a solution in $\mathcal{F}$, but we do not know if it has a corresponding element in $\mathcal{I}$ (and which fuzzy quantity it is). The complexity of this task depends only linearly on the number of variables, so solution of large systems need not be a problem. Nevertheless, in order to simplify the presentation, here we demonstrate it on the simplest examples.

\section{Examples of application}

In this section, $\vec{X}, \vec{Y}, \ldots \in \mathcal{F}$, etc. denote arbitrary free fuzzy quantities; they need not be from the cone $e(\mathcal{I})$, i.e., they need not correspond to any real fuzzy quantity from $\mathcal{I}$.

Example 14 Let us consider the following system of linear equations:

$$
\begin{aligned}
& 2 \cdot X+Y=A, \\
& X+2 \cdot Y=B,
\end{aligned}
$$

where fuzzy quantities $A, B$ are given and fuzzy quantities $X, Y$ are unknown. The embedding $e$ translates this to a system in $\mathcal{F}$ :

$$
\begin{aligned}
& 2 \odot \vec{X} \oplus \vec{Y}=\vec{A}, \\
& \vec{X} \oplus 2 \odot \vec{Y}=\vec{B},
\end{aligned}
$$

where $\vec{A}, \vec{B}, \vec{X}, \vec{Y} \in \mathcal{F}$. It has a unique solution in $\mathcal{F}$ :

$$
\begin{aligned}
\vec{X} & =\frac{1}{3} \odot(2 \odot \vec{A} \ominus \vec{B}), \\
\vec{Y} & =\frac{1}{3} \odot(2 \odot \vec{B} \ominus \vec{A}) .
\end{aligned}
$$

It can be rewritten in the previous form:

$$
\begin{aligned}
\vec{X} & =\frac{1}{3} \odot([(0,2 A)] \ominus[(0, B)]) \\
& =\frac{1}{3} \odot[(B, 2 A)] \\
& =\left[\left(\frac{1}{3} B, \frac{2}{3} A\right)\right], \\
\vec{Y} & =\frac{1}{3} \odot([(0,2 B)] \ominus[(0, A)]) \\
& =\frac{1}{3} \odot[(A, 2 B)] \\
& =\left[\left(\frac{1}{3} A, \frac{2}{3} B\right)\right],
\end{aligned}
$$

It remains to find the fuzzy quantities corresponding to $\vec{X}, \vec{Y} \in \mathcal{F}$ (if there are any), i.e., to solve equations

$$
\begin{aligned}
X+\frac{1}{3} B & =\frac{2}{3} A, \\
Y+\frac{1}{3} A & =\frac{2}{3} B,
\end{aligned}
$$

which are of the form (1) and can be solved as in Example 1.

The same procedure can be applied to more complex systems of equations.

\section{Systems with negative coefficients}

As Theorem 11 applies only to multiplication by positive scalars, we need another technique for dealing with negative coefficients. This is unnecessary in some special cases.

Example 15 To solve the system

$$
\begin{aligned}
& 2 \cdot X-Z=A, \\
& X-2 \cdot Z=B,
\end{aligned}
$$

where $X, Z$ are unknowns, it suffices to substitute $-Z=Y$ and we obtain system (4). Then we compute $Z=-Y$ according to the standard fuzzy arithmetic.

Problems occur when some unknown variable has coefficients with both signs. Then we need the operation $\sim$ in $\mathcal{F}$ which corresponds to unary minus $(-$, equivalently, multiplication by -1$)$ in $\mathcal{I}$ :

$$
\sim \vec{X}=\overrightarrow{-X}=[(0,-X)] .
$$

This is not the unary minus $(\ominus)$ in $\mathcal{F}$. To compare the two operations, we evaluate their difference:

$\ominus X \ominus(\sim \vec{X})=[(X, 0)] \ominus[(0,-X)]=[(X-X, 0)]$, which typically is not $\overrightarrow{0}$.

We defined the operation $\sim$ on the cone $e(\mathcal{I})$, but it is a symmetry which can be extended to the whole of $\mathcal{F}$ by

$$
\sim[(C, D)]=[(-C,-D)]
$$

Proposition 16 Properties of the operation on $\mathcal{F}$ :

$$
\begin{aligned}
\text { 1. } & \sim(\sim[(C, D)])=[(C, D)] \\
\text { 2. } & \sim(\ominus[(C, D)])=\ominus(\sim[(C, D)]), \\
\text { 3. } \sim([(A, B)] \oplus[(C, D)]) & \sim \sim[(A, B)] \oplus(\sim[(C, D)]) \\
& =\sim[(\sim \odot[(C, D)])=r \odot(\sim[C, D)]) .
\end{aligned}
$$

The solution of systems of linear equations with different signs of coefficients lead to simultaneous restrictions on vectors $\vec{X}, \sim \vec{X}$. The solutions are typically not unique. We leave detailed description of this problem to a future paper. 


\section{Conclusions and topics for further research}

We embedded fuzzy quantities into a vector space (with a different unary minus). This allows to find possible solutions of systems of linear equations in the larger vector space, using standard methods of linear algebra. Then it remains to find to each variable the corresponding fuzzy quantity (if it exists). This step is always the same for each variable, independently of the complexity of the original system of equations.

For particular cases like trapezoidal fuzzy intervals or triangular fuzzy numbers, simpler algorithms can be formulated.

It is desirable to extend the results to (systems of) non-linear equations, including multiplication and division of fuzzy quantities.

The same principle allows also an extension to more dimensions, i.e., to fuzzy vectors.

Acknowledgement 17 The author wants to acknowledge the inspiration by late Professor Dan Butnariu. This work was motivated by discussions with him years ago.

This research was supported by the Czech Ministry of Education under project MSM 6840770038.

\section{References}

[1] Kaufmann, A., Gupta, M.M.: Introduction to Fuzzy Arithmetic. Theory and Applications. International Thomson Computer Press, London, 1991.

[2] Klir, G.J., Yuan, B.: Fuzzy Sets and Fuzzy Logic. Theory and Applications. Prentice-Hall, 1995.

[3] Klir, G.J.: The role of constrained fuzzy arithmetic in engineering. In: B.M. Ayyub and M.M. Gupta (eds.), Uncertainty Analysis in Engineering and Sciences: Fuzzy Logic, Statistics, and Neural Network Approach, Kluwer, Dordrecht, 1997, 1-19.

[4] Klir, G.J., Pan, Y.: Constrained fuzzy arithmetic: Basic questions and some answers. Soft Computing 2 (1998), No. 2, 100-108.

[5] Mareš, M.: Computation over Fuzzy Quantities. CRC Press, Boca Raton, 1994.

[6] Navara, M., Žabokrtský, Z.: How to make constrained fuzzy arithmetic efficient. Soft Computing 5 (2001), No. 6, 412-417. 